\section{Dichoptic interaction of visual structure and light flashes*}

\author{
P. ESKILDSEN, T. S: NEWTON, and D. LEHMAN† \\ Smith-Kettlewell Institute and Department of Visual Sciences \\ University of the Pacific, San Francisco, California 94115
}

Perceptual interaction between monocular flashes and different amounts of continuously visible target structure shown to the other eye was investigated. A response variable called dominance of the flashes is defined and found to be a direct function of flash intensity and an inverse function of contraocular target structure. The dominance variable is distinguished from subjective brightness of the flashes and subjective complexity of the targets. The observed effects are attributed to limited central processing capacity with increased informational load.

The presentation of visual structure to one eye has been shown to degrade the percept received via the other eye (Flom et al, 1963; Schiller, 1965). Also, the electrical power of average EEG potentials evoked by monocular light flashes of constant parameters is reduced by contraccular presentation of continuously illuminated visual structure (Lehmann \& Fencer, 1968); in these experiments, Ss had been instructed to fixate on the structured target. The authors suggest that the effect is central, resulting from an increase in informational loading of binocular elements by the structured field which leaves less capacity available for rexponse to light flashes presented to the other eye.

With increased contreocular structure, some of Lehmann and Fender's Ss reported a decrease in flash brightnes. These subjective reports suggested brightness as a posible paychological correlate to the obeerved changes in the evoked potential. A series of preliminary experiments was conducted to measure the judged brightness of the flashes as a function of contraccular target structure. The results of these experiments were consistently negative in that S's brightness estimates were not affected by structure. This was true whether or not $S$ was specifically instructed to fixate on the structure target, rather than the flashes, while making brightness judgments of the flash trains.

Although the brightness estimates were not influenced by contraocular structure, a striking perceptual effect is seen in the dichoptic stimulus condition, best described as the impressiveness or force of the flash. With greater flash luminance or less contraocular structure, the flashes have more strength, more impact, and are

- Supported by USPHS, NIH, Grant NB-06038. †Present address: Department of Neurology, University Hospitals, Zurich, Switzerland. unpleasant. We call this effect the "dominance" of the flashes. This use of the word, dominance, is descriptive and does not imply the presence of mechanisms active in binocular rivalry or masking experiments. We investigated the effect of monocular flash luminance and contraccular steady target structure on subjective dominance of the flashes.

\section{METHOD}

Six paid Ss, ages 17 to 25 , with at least high school education, served in one expeximental session each. The display apparatus was located in a sound-shielded dark room (Industrial Acoustics) equipped with an intercom system for communication with E. A modifed Wottring troposcope was used to present the two subjectively superposed circular fields of $20 \mathrm{deg}$ visual angle. The field for the right eye was uniformly illuminated by 3.7 flashes per second, generated by a sound-shielded Grass PS 2 xenon stroboscope with the nominal relative intensity settings of $1,2,4,8$, and 16 . Flash duration was less than 10 microsec; at Setting 16 the lamp produced 1.5 million candle power (according to the manufacturer's specification), which was attenuated by a factor of 1,000 in our optical system. Settings of $1,2,4$, and 8 were used in our study. In each trial, one of five targets was presented to the left eye in a field continuously illuminated by $3 \mathrm{~mL}$. In order of increasing structure, the targets were: (1) a blank field, (2) a centered black dot, (3) a line cross, (4) a horizontal-vertical grid pattem with 7 lines evenly spaced to cover the target field, (5) a similar grid pattern with 15 lines. The dot and the lines subtended 5 deg of visual angle. Only the 15-line grid measurably altered the mean target luminance, reducing it by the equivalent of .15 density units.

The five targets with the four flash intensities formed a block of 20 stimulus combinations, which were shown to the $S$ in a random sequence. Three differently randomized blocks of 20 trials were presented, resulting in three observations per stimulus combination.

Upon entering the experimental room, $\mathrm{E}$ informed $S$ of the general nature of the experiment, adjusted the apparatus to S's head, and read the instnuctions. A 15-min dark-adaptation period preceded the data collection. Normal pupils were used, since dilation of the right pupil had not yielded different results in preliminary studies. The duration of each stimulus presentation was $10 \mathrm{sec}$, followed by a $30-\mathrm{sec}$ intertrial interval, during which $S$ reported his judgment and changed the target according to E's instructions. The total time for a session was about $45 \mathrm{~min}$.

\section{INSTRUCTIONS}

The Ss were shown four stimulus combinations to familiarize them with the task. They were asked to fixate the center of the target and to observe the extent to which the flashes dominated the field. Among the four stimulus combinations were flashes (at Setting 8) shown with a dark contraocular field. The dominance of this combination was assigned the value of 100. The Ss were then instructed to judge the dominance of the flashes, in each case proportionally to the value of the standard, and were given practice using the other three stimulus combinations.

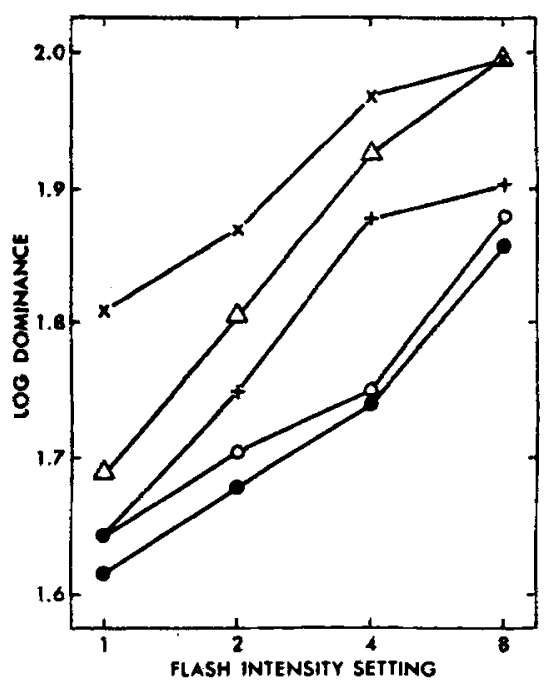

Fig. 1. Log dominance of the flashes over the target, for each of the five targets: blank $(X)$, dot $(\Delta)$, cross $(+), 7$-line grid $(0)$, 15-line grid $(*)$. Scaled judgments are averaged over all Ss. Values obtained with same target are connected by lines. The absciss represents intensity of the flashes on an ordinal scale. 


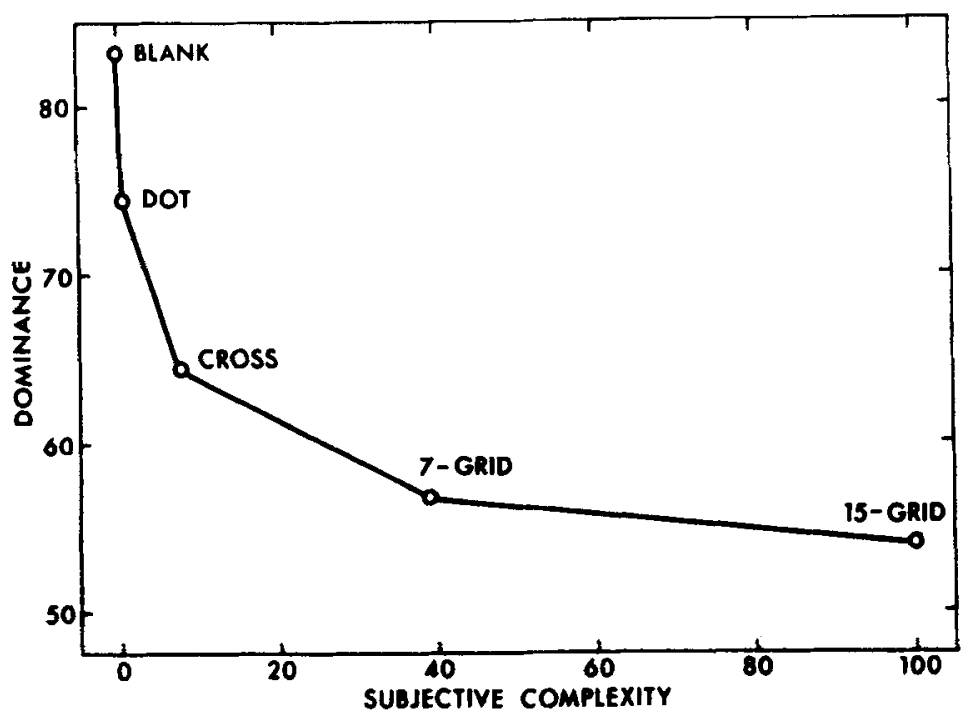

Fig. 2. Relative dominance of the flashes over the structure target as a function of judged complexity of the targets.

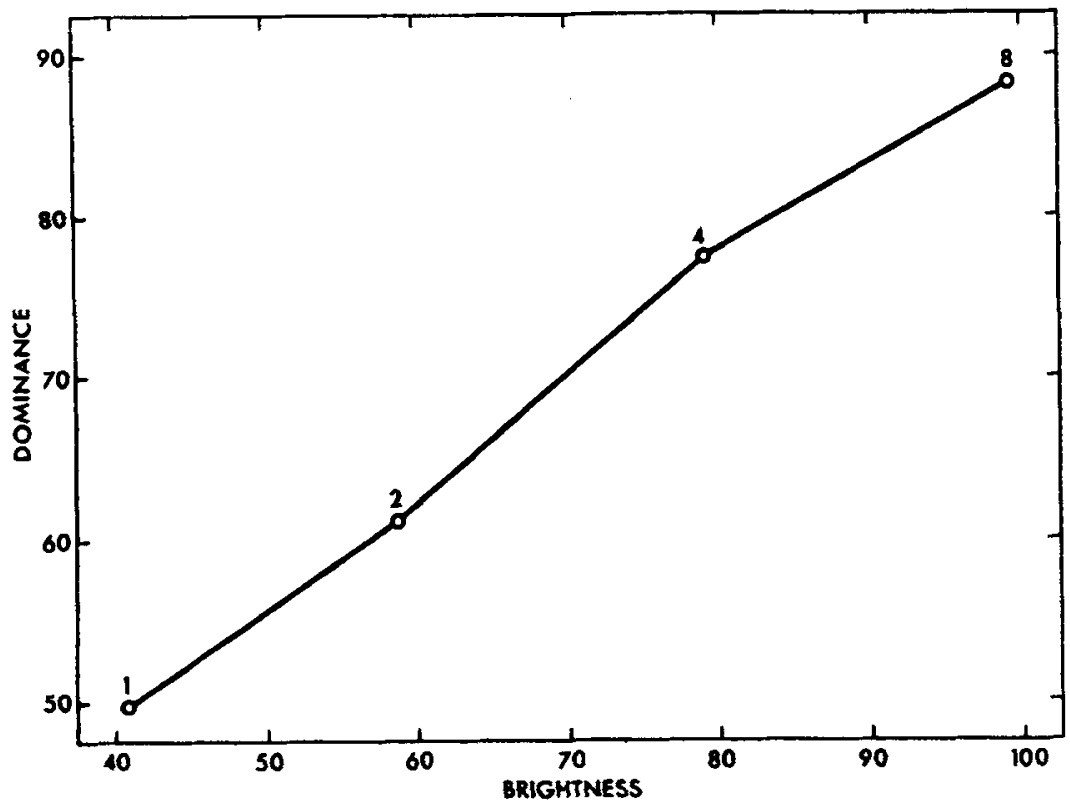

Fig. 3. Relative dominance of the flashes over the structure target as a function of brightness, for each of the four intensity settings $(1,2,4,8)$ used.

\section{RESULTS}

The three judgments obtained for each stimulus combination were averaged for each $S$. The judgments of each $S$ were multiplied by a constant which gave a mean value of 100 for the stimulus combination (Intensity Setting 8 , blank field). The transformed values were then averaged over Ss and plotted in Fig. 1. As can be seen, perceptual dominance increases with increasing flash intensity. There is an inverse relationship between amount of structure and judged dominance of the flashes.

A three-factor analysis of variance (4 levels of intensity, 5 targets, 6 Ss, 3 observations per cell) was performed on the raw data. The intensity and target variables were treated as fixed effects, Ss as random effects variables. The $F$ ratios for both intensity and target main effects were significant beyond the .005 level, but the significance of interaction between these variables reached only the .05 level.

\section{DISCUSSION}

Analysis of the data indicates that dominance can be considered as having two components, one resulting from the effects of intensity of the flashes, the other resulting from the effects of target structure which is shown to the contralateral eye.

One might speculate that the reduction in dominance by increased target structure results from Ss' rating complexity of the target structure and using the complexity rating as a basis for the dominance judgment. If this were the case, a linear relationship between judged complexity of the targets and dominance would be expected. To test this hypothesis, a separate experiment, using a second group of $27 \mathrm{Ss}$, was performed. A magnitude estimation scale was made of the complexity of the five targets used in the main experiment. The results of wis separate experiment are shown in Fig. 2 , which exhibits relative dichoptic flash dominance as a function of judged target complexity. Obviously, dominance and complexity are not related by a linear function.

However, judged dominance appears to have a linear relationship with judged brightness under our experimental conditions, as shown in Fig. 3. This implies that both dominance and brightness increase systematically and in similar fashion with increased intensity of the flashes. On the other hand, dominance, unlike brightness, is decreased systematically by increase in contraocular structure.

Our experiment describes a perceptual dimension for which an electroneurophysiological correlate has been reported and interpreted as change in available neural capacity (Lehmann \& Fender, 1968, 1969). The observed change of size of the evoked potentials had pointed to a possible perceptual change in brightness, but if the $S$ is asked to rate brightness, manipulation of structure is ineffective. Thus, our dominance effect cannot be attributed to mechanisms of interocular contour and brightness interaction described by Levelt (1968). Apparently, when asked to rate brightness of the flashes, Ss' judgments are influenced only by the flash luminence, but not by the structure of the contralateral target. The required judgment of dominance, on the other hand, implies the simultaneous consideration of both flashes and structure targets. Therefore, the necessity of parallel processing of the two inputs by the same system seems to be the basis of the observed perceptual interaction between the monocular light flashes and the amount of contraocular structure.

\section{REFERENCES}

FLOM, C., HEATH, C. G., \& TAKAHASHI, E. Contour interaction and visual resolution: Contralateral effects. Science, 1963, 142, 979-980.

LEHMANN, D., \& FENDER, D. H. Component analysis of human averaged evoked potentials: Dichoptic stimuli using different target structure. Electroencephalography \& Clinical Neurophysiology, 1968, 24, 542-553. 
LEHMANN, D., \& FENDER, D. H. Averaged visual evoked potentials in humans: Mechanism of dichoptic interaction studied in a subject with a split chiasm. Electroencephalography \& Clinical
Neurophysiology, 1969, 27, 142-145.

LEVELT, W. J, M. On binocular rivaly. The Hague: Mouton, 1968.

SCHILLER, P. H. Monoptic and dichoptic visual masking by pattern and flashes. Journal of
Experimental Psychology, 1965, 69, 193-199.

(Accepted for publication October 1, 1970.) 